\title{
BK Virus Reactivation
}

National Cancer Institute

\section{Source}

National Cancer Institute. BK Virus Reactivation. NCI Thesaurus. Code C160245.

The switching of latent BK virus to a lytic infection. 